\title{
Comparación de Modelos Empíricos de pérdida LPWAN por Propagación en el campus Prosperina de la ESPOL: Resultados derivados de un estudio empírico
}

\section{LPWAN Empirical Propagation Path Loss Models comparison at Prosperina campus in ESPOL: Results based on an empirical study}

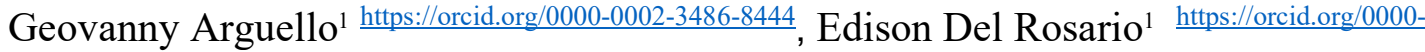 \\ $\underline{0003-4573-2048}$, Edison Boris Ramos ${ }^{1}$ https://orcid.org/0000-0002-0469-6135
}

'Escuela Superior Politécnica del Litoral - ESPOL, Guayaquil, Ecuador geomaarg@espol.edu.ec, edelros@espol.edu.ec, bramoseespol.edu.ec

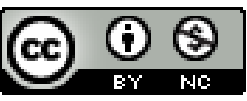

Esta obra está bajo una licencia internacional Creative Commons Atribución-NoComercial 4.0
Enviado: $\quad 2021 / 06 / 11$

Aceptado: $\quad 2021 / 09 / 28$

Publicado: $\quad 2021 / 11 / 30$

\section{Resumen}

La tecnología de largo alcance LoRa, como parte de tecnologías de bajo consumo energético LPWAN, permite establecer comunicaciones inalámbricas a kilómetros de distancia con bajas tasas de transmisión. Sus características, inducen a la creación de nuevas líneas de investigación para comprender su comportamiento en diversos ambientes y diferenciarlas de otras tecnologías similares. En esta investigación, se caracteriza a la tecnología LoRa en contraste con Sigfox y NBIoT. Se evalúa el efecto de pérdidas en la propagación que tiene la tecnología LoRa, con mediciones realizadas en ambientes tanto abiertos como cubiertos por vegetación, dentro de las instalaciones de la Escuela Superior Politécnica del Litoral (ESPOL), campus Gustavo Galindo Velasco o Prosperina. Se establece un modelo de pérdida por gradiente, utilizando regresión de mínimos cuadrados (LS). Se registra el parámetro RSSI en paquetes enviados a través de una topología punto a punto entre nodos, y se obtiene un valor de potencia promedio recibida en cada nodo. El modelo obtenido, es de gradiente múltiple y se lo compara con otros modelos de propagación como el modelo de pérdida en espacio libre (FSL), y tres modelos empíricos como son, modelo COST-231 Hata, modelo de la ciudad de Dortmund (Alemania) y el modelo de la ciudad de Oulu (Finlandia), siendo este último el que presenta menor error de dispersión con el modelo obtenido.

Palabras clave: gradiente, LoRa, LPWAN, modelo de propagación, regresión de mínimos cuadrados (LS).

Sumario: Introducción, Diferencias técnicas y factores IoT: LoRa, Sigfox y NBIoT, Metodología, Modelos empíricos, Resultados y discusión y Conclusiones.

Como citar: Arguello, G., Del Rosario, E. \& Ramos, E. (2021). Comparación de Modelos Empíricos de pérdida LPWAN por Propagación en el campus Prosperina de la ESPOL: Resultados derivados de un estudio empírico. Revista Tecnológica - Espol, 33(2), 12-24.

http://www.rte.espol.edu.ec/index.php/tecnologica/article/view/824 


\begin{abstract}
Long-range technology LoRa, as part of low power consumption technologies LPWAN, allows wireless communications within kilometers of distance using low data rates. Its characteristics induce the creation of new lines of research to have a better understanding of its behavior in different environments and differentiate it from other similar technologies. In this research, LoRa technology was characterized by the contrast of Sigfox and NBIoT. It was evaluated the path loss effect in LoRa technology, using measurements in open environments and forest environments, at Prosperina Campus - ESPOL. A path loss gradient model using Least Squares Regression (LS) was found. RSSI parameter was registered, from sending packages through point-to-point node's topology and getting a mean power from each node. The multi-gradient model obtained was compared with other propagation models such as the Free Space Loss (FSL) model and three empiric models: COST-231 Hata model, model of Dortmund city (Germany), and model from Oulu city (Finland). Among these models, the latter presented the lowest dispersion error regarding the obtained model.
\end{abstract}

Keywords: gradient, LoRa, LPWAN, propagation model, least square regression.

\title{
Introducción
}

En la actualidad, junto con el desarrollo del Internet de las cosas (IoT), existen cada vez más dispositivos intercomunicados entre sí (Kais Mekki, 2018). Una de las tecnologías de comunicación que ha ganado popularidad en los últimos tiempos, son aquellas conocidas como LPWAN (Low Power Wide Area Network), debido a su largo alcance y bajo consumo energético. Lora, Sigfox y NBIoT son tres de las tecnologías LPWAN más utilizadas para el desarrollo de aplicaciones IoT dependiendo las características que se requieran.

Los modelos de propagación ayudan en la planificación y despliegue de redes, particularmente para realizar estudios durante el despliegue inicial y la viabilidad del proyecto. También se los utiliza en estudios para calcular la medida de la interferencia conforme avanza el despliegue de la red. En las redes inalámbricas, se pueden caracterizar tres tipos de modelos; estos son: empíricos, determinísticos y estocásticos (V.S. Abhayawardhana, 2005). Los modelos empíricos son los que se basan únicamente en observaciones y mediciones. Estos modelos se utilizan principalmente para predecir la medida de pérdida por trayectoria, pero también existen modelos que predicen el desvanecimiento por lluvia y la trayectoria múltiple. Los modelos deterministas hacen uso de las leyes que gobierna la propagación de ondas electromagnéticas para determinar la potencia de la señal recibida en una ubicación particular.

Los modelos deterministas, a menudo requieren un mapa completo en 3D de la propagación ambiente. Un ejemplo de modelo determinista es un modelo de trazado de rayos. Los modelos estocásticos, por otro lado, modelan el medio ambiente como una serie de variables aleatorias. Estos modelos son los menos precisos, pero requieren la menor información sobre el medio ambiente y utilizan menos potencia de procesamiento para la generación de predicciones. Los modelos empíricos se pueden dividir en dos subcategorías, disperso en el tiempo y no disperso en el tiempo. El primero, está diseñado para proporcionar información relacionada con las características temporales que sufre el canal de propagación usado; es decir, el retardo por múltiples trayectorias en la propagación del canal. Un ejemplo de este tipo son los que se desarrollaron en la Universidad de Stanford (SUI), con un grupo de trabajo conformado por personal del Instituto de Ingenieros Eléctricos y Electrónicos (IEEE) 802.16. Ejemplos de modelos empíricos no dispersivos en el tiempo son ITU-R, Hata y el modelo COST-231 Hata (Hendrik Linka, 2018). Todos estos modelos predicen la media de la pérdida por trayectoria en función de varios parámetros, por ejemplo, distancia, alturas de antena, etc. 
Aunque los modelos de propagación empíricos para sistemas móviles se han validado de forma exhaustiva, su idoneidad para ser considerados estándares, no ha sido completamente establecida. Este artículo propone un modelo empírico de propagación basado en mediciones realizadas en el campus de la ESPOL, ayudado de análisis estadístico para calcular el gradiente-pendiente resultante, tanto en ambientes cubiertos con vegetación, como en ambientes abiertos. Luego, se compara con varios estudios empíricos presentados y expuestos en la comunidad científica. Se analiza el efecto de la línea de vista en la tecnología LoRa.

\section{Diferencias técnicas y factores IoT: LoRa, Sigfox y NBIoT}

A pesar de que LoRa, Sigfox y NBIoT son consideradas tecnologías LPWAN, cada una de ellas presenta características propias dadas por el fabricante:

\section{LoRa}

Es una tecnología de capa física, que utiliza una técnica de ensanchamiento del espectro, de origen propietario por la compañía SEMTECH, para la transmisión o recepción de datos. El rango de frecuencias para la modulación está comprendido entre $860-960 \mathrm{MHz}$ para Europa y América, y frecuencias de $400 \mathrm{MHz}$ para Asia. Admite comunicación bidireccional a través de la modulación por "chirp" chirrido de ensanchamiento del espectro (CSS), metiendo una señal mensaje en un ancho de banda angosto (Ayanle I. Ali, 2019).

Para el mejor entendimiento del CSS, se utilizan conceptos como chip y chirp. Un chip equivale a una medida de cantidad dentro de un tiempo fijo, equivalente al ancho de banda utilizado para transmitir símbolos; es decir, si se tiene un ancho de banda de $250 \mathrm{khz}$, entonces, se tiene 250000 chips por segundo. La cantidad de chips que pueden ser utilizados para la creación de un símbolo viene dada por el factor de esparcimiento (SF), y se rige a la siguiente fórmula: $2^{S F}$, el SF también indica la cantidad de bits que se utilizarán para un símbolo. El SF va de 7 a 12, mientras mayor el SF, mayor es la distancia de cobertura y menor la tasa de transmisión; este efecto es debido a que aumenta la cantidad de chips por símbolo. Por otro lado, un chirp no es nada más que la rampa utilizada para la creación de símbolos, que puede ser 'up-chirp' o 'down-chirp'.

La Figura 1 muestra diferentes símbolos de una trama LoRa, detallando características intrínsecas de esta tecnología.

\section{Figura 1}

Diferentes simbolos LoRa, la primera mitad con 'up-chirp' y el restante con 'down-chirp'

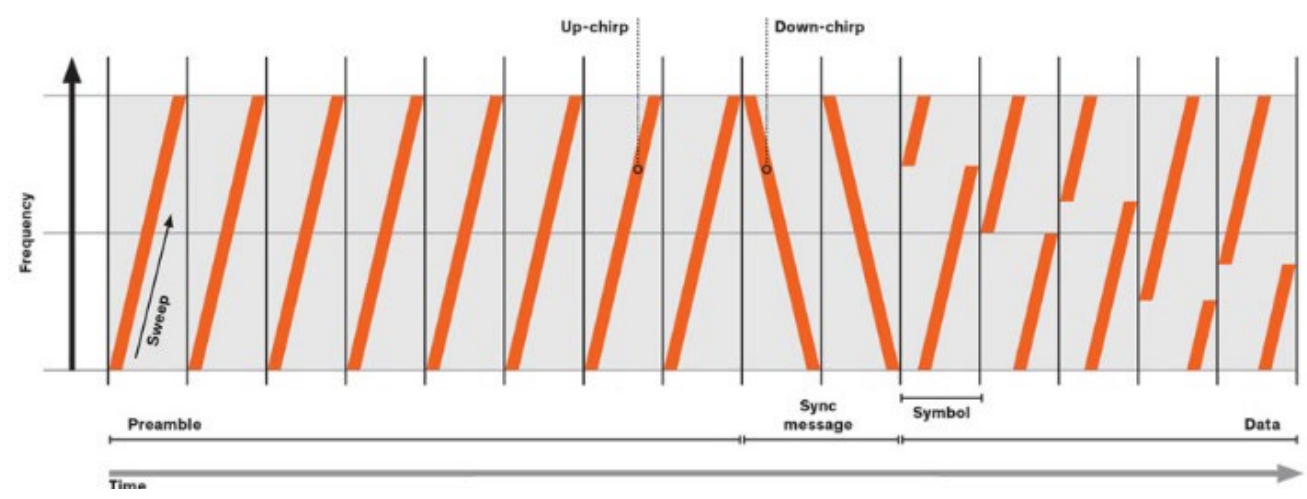

Los conjuntos de todas estas características de la señal resultante permiten reducir las interferencias y llegar a largas distancias. Lora adicionalmente utiliza un método de corrección 
de errores (FEC) con tasas de codificación 4/5, 4/6, 4/7 y 4/8; para mejorar la sensibilidad en la recepción (Usman Raza, 2017).

\section{Sigfox}

Tecnología que usa modulación por desplazamiento de fase binaria (BPSK) sobre una portadora muy angosta (UNB) de aproximadamente $100 \mathrm{~Hz}$. El uso de UNB mejora la sensibilidad en el receptor ya que se utiliza de manera eficiente el espectro, generando poco consumo energético. Todo esto suma para tener una tasa de datos máxima de 100 bits por segundo. Una de las desventajas que presenta Sigfox es la limitación en el tamaño del mensaje a transmitir y la cantidad de veces por día tanto para subida como para bajada (Usman Raza, 2017).

\section{NBIoT}

Tecnología que coexiste con la tecnología LTE, por lo tanto, depende de una infraestructura presente. Utiliza modulación por desplazamiento de fase en cuadratura (QPSK) junto con acceso múltiple por división de frecuencia (FDMA), ortogonal para descargas (DL) y única portadora para cargas (UL). Puede operar en 3 modos diferentes, único portador, en banda (compartiendo ancho de banda con portadora LTE) y banda de guarda (en una portadora ya existente). Puede alcanzar tramas hasta 1600 bytes con una tasa de transmisión de $20 \mathrm{kbps}$ para subida y 200 kbps para bajada (Massimo Ballerini, 2020).

La Tabla 1 muestra un resumen de las características tecnológicas existentes en LPWAN.

Tabla 1

Características tecnológicas de tecnologías (Oratile Khutsoane, 2017)

\begin{tabular}{|c|c|c|c|}
\hline & Lora & Sigfox & NBIoT \\
\hline Modulación & CSS & $\begin{array}{l}\text { UNB DBPSK(UL), } \\
\text { GFSK(DL) }\end{array}$ & QPSK \\
\hline Frecuencias & $\begin{array}{c}430 \mathrm{MHz} \& 860-960 \\
\mathrm{MHz}\end{array}$ & $868 \mathrm{MHz} \& 902 \mathrm{MHz}$ & Frecuencias LTE \\
\hline Rango & $<20 \mathrm{~km}$ & $<40 \mathrm{~km}$ & $<10 \mathrm{~km}$ \\
\hline Tasa de transmisión & $\begin{array}{c}0.3-37.5 \mathrm{kbps}(\mathrm{LoRa}) \\
50 \mathrm{kbps}(\mathrm{FSK})\end{array}$ & $\begin{array}{c}100 \text { bps (UL), } 600 \text { bps } \\
\text { (DL) }\end{array}$ & $\begin{array}{c}20 \mathrm{kbps} \text { (UL), } 200 \mathrm{kbps} \\
\text { (DL) }\end{array}$ \\
\hline Número de canales & $\begin{array}{c}10 \text { en EU, 64+8(UL) y } 8 \\
\text { (DL) en US, diferentes } \\
\text { SF }\end{array}$ & 360 canales & $\begin{array}{l}\text { Dependiendo el número } \\
\text { de bloques LTE }\end{array}$ \\
\hline Sincronismo & Sí & No & Sí \\
\hline FEC & Sí & No & Puede usar \\
\hline Tamaño de paquetes & Hasta 250 bytes & $\begin{array}{c}12 \text { bytes (UL), } 8 \text { bytes } \\
\text { (DL) }\end{array}$ & Hasta 1600 bytes \\
\hline Topología & Estrella - Multi estrella & Estrella & SEA \\
\hline
\end{tabular}

\section{Factores IoT}

Los factores principales de una tecnología para pertenecer a una red LPWAN son la distancia en la cobertura y el bajo consumo energético. Sin embargo, para satisfacer las necesidades de una aplicación IoT adicionalmente debe comprender factores como la calidad de servicio (QoS), tiempo de vida de la batería, latencia, escalabilidad, tamaño de la trama, modelo de desarrollo y costo. 


\section{Rango y cobertura}

A nivel de rangos Sigfox tiene ventaja sobre las demás, debido a que una sola estación base puede llegar a cubrir alrededor de $40 \mathrm{~km}$ a la redonda; por otro lado, Lora ofrece alcances de hasta $20 \mathrm{~km}$, mientras que NBIoT depende mucho de donde se encuentre una red LTE y sus alcances son menores a $10 \mathrm{~km}$ (Elmustafa Sayed Ali Ahmed, 2019).

\section{Tiempo de batería y latencia}

El consumo energético de las tres tecnologías es bajo, sin embargo, al tener una comunicación sincrónica, los picos de consumo de la tecnología NBIoT son mayores que las otras dos. La compensación para esto, es la latencia. Lora, a pesar de que posee dispositivos de clase $\mathrm{C}$ que son especializados para reducir la latencia aumentando el consumo energético, no llega a reducir la latencia tanto como con dispositivos NBIoT. Por lo tanto, para aplicaciones de poco envío de datos es recomendable usar Lora o Sigfox, pero para aplicaciones donde la latencia juega un papel importante se recomienda NBIoT.

\section{Calidad de servicio}

Lora y Sigfox utilizan bandas no licenciadas y protocolos de comunicación asíncronos, los mismos que son utilizados para mitigar los efectos de interferencias como co-canal, caminos múltiples y desvanecimiento. Sin embargo, no llegan a brindar la misma calidad de servicio que da NBIoT, la misma que utiliza una banda licenciada y un protocolo síncrono basado en LTE. Para el uso de una banda licenciada, se tiene que tener en cuenta el costo para la adquisición/alquiler de la misma, que depende de la región donde se la utilice (Gutierrez, 2019).

\section{Escalabilidad y longitud de trama}

LoRa, Sigfox y NBIoT soportan una gran cantidad de nodos conectados a una estación base; no obstante, NBIoT permite mayor escalabilidad ya que teóricamente admite hasta 100 mil usuarios por celda en comparación con LoRa y Sigfox que admiten hasta 50 mil. Así mismo, la longitud de trama es mayor para aplicaciones con tecnología NBIoT, hasta 1600 bytes, seguidos por LoRa, hasta 243 bytes y finalmente Sigfox con un máximo de 12 bytes para transmisión, lo que limita mucho en el uso de aplicaciones.

\section{Costo y licenciamiento}

NBIoT no tiene un modelo de red establecido, ya que utiliza o comparte infraestructura de red LTE, mientras que Sigfox y LoRa son tecnologías actualmente en comercialización y que poseen diferentes aplicaciones alrededor del mundo. Una de las ventajas significativas de los ecosistemas LoRa es la flexibilidad, ya que admiten desarrollos locales, utilizando Gateways LoRa, y desarrollos públicos usando estaciones bases. Este modelo híbrido permite tener mejor acogida en campos industriales. Para proyectar el costo en una red LPWAN, es necesario entender la necesidad de pagar o no por el espectro donde se usa, la estación base a utilizar y el costo de un nodo en particular. NBIoT utiliza el espectro licenciado, el mismo que tiene un costo dependiendo la cantidad de ancho de banda a utilizar en su respectivo país. LoRa y Sigfox utilizan una banda no licenciada por lo que no hay costos por la utilización del espectro. Adicionalmente, el costo por estación base o Gateway Sigfox es mayor a un Gateway Lora, ya que existen en la actualidad gateways de bajo presupuesto para desarrollo que se conectan con hardware ya existente como la Raspberry Pi. Finalmente, están las estaciones base NBIoT, cuyo costo excede a otras tecnologías debido a la necesidad de establecer una red celular LTE para su uso. En cuanto a los nodos o dispositivos finales, el costo entre LoRa o Sigfox es muy similar, siendo los dispositivos NBIoT los más costosos en el mercado (Luis F. Ugarte, 2019) (Juha Petäjäjärvi, 2015). 
Figura 2 muestra una sinopsis de cada tecnología versus su factor IoT mencionado. La tecnología LoRa es presentada en color azul, Sigfox en color naranja y NBIoT en amarillo. Cada uno de estos parámetros permiten tener una mejor imagen global con respecto a la aplicación que se desee realizar.

\section{Figura 2}

\section{Comparación entre factores IoT}

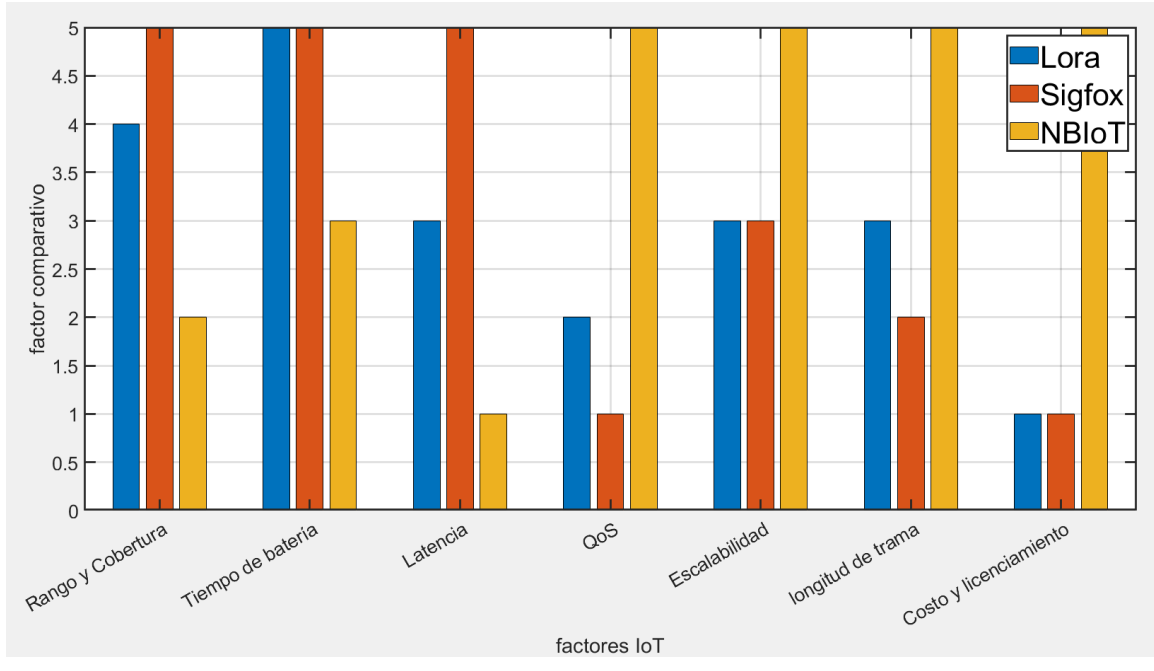

\section{Metodología}

Una red basada en dos nodos fue establecida dentro del campus Gustavo Galindo Velasco o Prosperina de la Escuela Superior Politécnica del Litoral. Uno de estos fue colocado de forma estática en la parte superior del edificio de la Facultad de Ingeniería Eléctrica de Ingeniería en Electricidad y Computación (FIEC). Para la identificación del nodo estático, se atribuyó un nombre conforme a la ubicación de la facultad donde se encuentra. Nodo FIEC fue el nombre elegido, y su ubicación geográfica viene referenciada con -2.144914 de latitud y -79.967758 de longitud. La Figura 3 muestra en vista aérea las instalaciones de la ESPOL, georreferencia la ubicación del nodo estático FIEC y de los puntos donde fueron tomadas las pruebas.

La altura del Gateway FIEC, con respecto a la superficie del terreno de la universidad, es de 22 metros.

El otro nodo fue utilizado como un nodo móvil, con movimiento dentro del área de cobertura del nodo estático tanto en ambientes abiertos, como en ambientes cubiertos por vegetación, esto debido al entorno encontrado durante el recorrido y toma de mediciones, el mismo que empezaba en un lugar denso y cubierto por vegetación, hasta un lugar más abierto, en ciertos casos obstruido por edificios. La elevación del nodo móvil fue de 0.70 metros sobre la superficie de la universidad, para simular un ambiente real.

Para cada punto de medición móvil, se utilizó una unidad de GPS diferencial para establecer la correcta ubicación del punto. Todas las mediciones se realizaron a una frecuencia de $915 \mathrm{MHz}$, con un ancho de canal de $20 \mathrm{kHz}$ y un factor de esparcimiento de 10. Para esta investigación se tomaron mediciones en 22 puntos diversos, diferenciados por circuitos, uno llamado FIEC (dentro de la vegetación) y el otro circuito llamado FCNM, por la Facultad de Ciencias Naturales y Matemáticas de la ESPOL, en campo abierto/suburbano mostrado en las Figuras 4(a) y 4(b), respectivamente. En cada punto de medición georreferenciado se 
establecieron 100 muestras, de las cuales se calculó el RSSI promedio y el SNR. Las ganancias utilizadas para las antenas fueron de $3 \mathrm{dBi}$ para el nodo estático y $0 \mathrm{dBi}$ para el nodo móvil. El valor de la intensidad de señal recibida (RSSI) fue utilizado para hallar el gradiente (pendiente) del modelo, mientras que el valor de potencia recibida fue utilizado para la estimación de la pérdida por propagación (pathloss) en cada punto de medición.

\section{Figura 3}

Ubicación en mapa del nodo estático FIEC

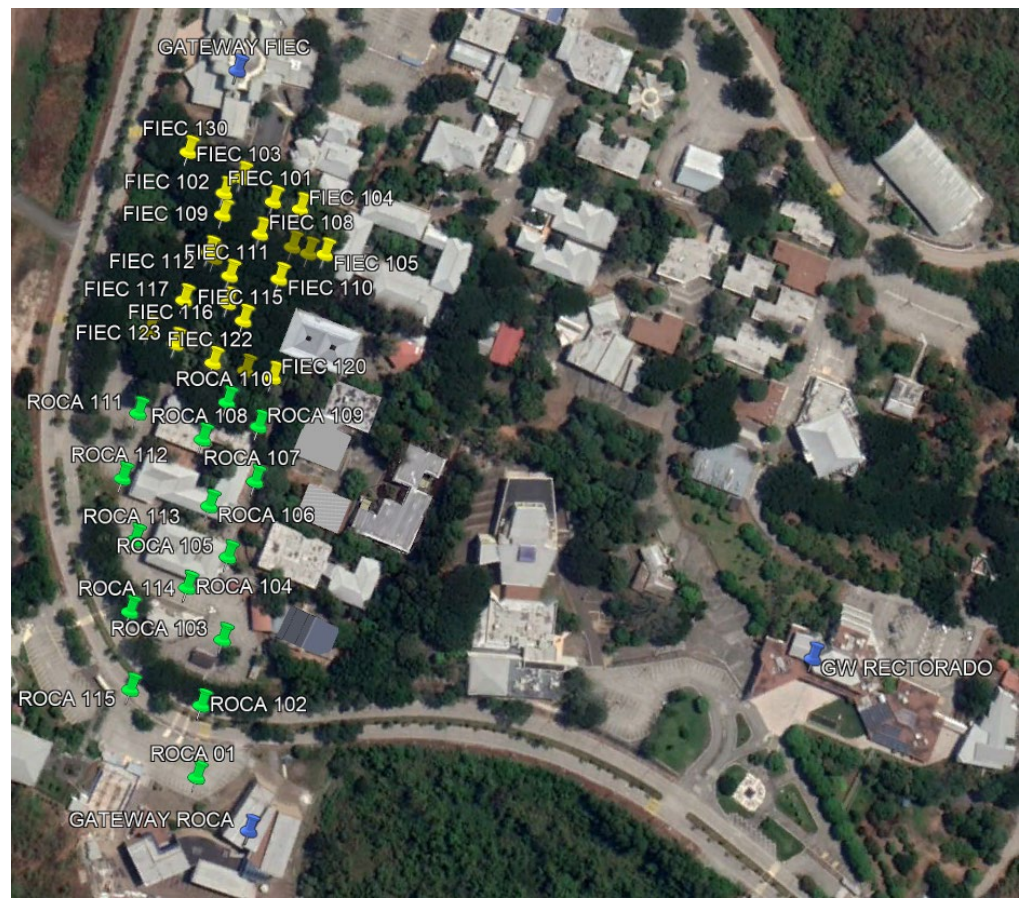

Figura 4

Ambientes de medición: a) Circuito FIEC (con vegetación) b) Circuito FCNM (abierto/suburbano)
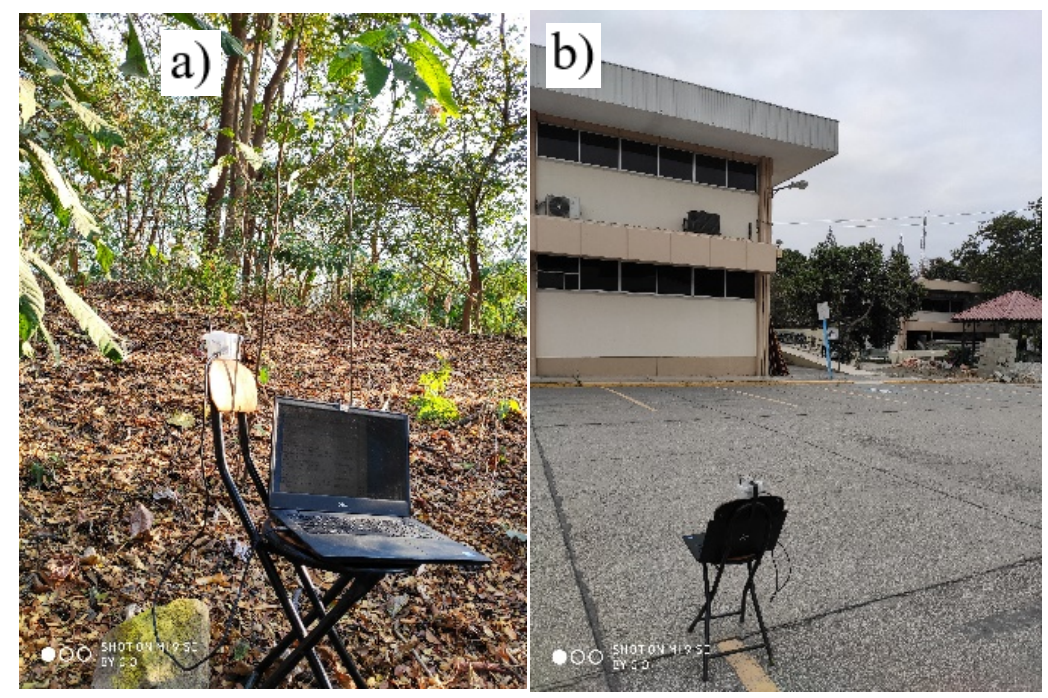

La pérdida por propagación puede ser definida como la distancia entre la potencia transmitida en función de la recibida, usualmente expresada en decibelios. La ecuación para el análisis de regresión de los mínimos cuadrados (LS) es: 


$$
P L(d)=P L\left(d_{0}\right)+10 n \log _{10}\left(\frac{d}{d_{0}}\right)
$$

Donde, $d_{0}$ es el punto de referencia a 1 metro de distancia del nodo, y $\mathrm{n}$ es conocido como el exponente de pérdida de propagación. Los valores de PL son expresados en decibelios. Para el cálculo de la pérdida en espacio libre (FSL) n es igual a dos. La importancia del valor de $\mathrm{n}$ es la rapidez con la que decae la potencia con respecto a la distancia.

Para la obtención de un modelo estadístico significativo utilizando el método de regresión de los cuadrados mínimos, se juntaron mediciones con ambientes similares. No se incluye una discriminación con los puntos que tienen línea de vista y los que no (LOS \& NLOS).

\section{Modelos empíricos}

Los resultados del modelo gradiente obtenido, utilizando el método de regresión de los mínimos cuadrados, será comparado con otros modelos empíricos aceptados en la actualidad. Se evaluarán tres modelos conocidos, modelo COST-231 Hata, modelo Oulu y el modelo Dortmund (V.S. Abhayawardhana, 2005). Adicionalmente se tomará como guía la pérdida en espacio libre (FSL).

\section{Modelo por pérdidas en espacio libre (FSL)}

Modelo que sirve como guía para nuestro análisis, basado en un cálculo puramente analítico, el FSL (ecuación de Friis) se calcula:

$$
P L(F S L)=20 \log _{10}(f)+20 \log _{10}(d)+32.45
$$

Donde d es la distancia en $\mathrm{km}$, f es la frecuencia de operación del sistema expresada en $\mathrm{MHz}$, y las unidades del modelo son en decibelios (dB).

\section{Modelo Cost-231 Hata}

Modelo altamente usado para predecir pérdidas por propagación en sistemas inalámbricos. Es un modelo basado en el modelo Okumura-Hata (Commission), 1999), pero con un factor de corrección indicado para trabajar en un rango de frecuencias entre $500 \mathrm{MHz}$ y $2000 \mathrm{MHz}$. Este factor de corrección se aplica para ambientes urbanos, sub-urbanos y rurales, dependiendo el caso. La ecuación básica para la pérdida de propagación con este modelo, viene dada por:

$$
\begin{array}{r}
P L(\text { Cost })=46.3+33.9 \log _{10}(f)-13.82 \log _{10}\left(h_{b}\right)-a h_{r}+ \\
\left(44.9-6.55 \log _{10}\left(h_{b}\right)\right) \log _{10}(d)+c m
\end{array}
$$

Donde, $\mathrm{f}$ es la frecuencia en $\mathrm{MHz}$, d es la distancia en $\mathrm{km}$ y $h_{b}$ es la altura de la antena estática sobre el nivel del suelo, en metros. El parámetro $\mathrm{cm}$ es igual a $0 \mathrm{~dB}$ para ambientes sub-urbanos o abiertos; mientras que toma el valor de 3 para ambientes urbanos. El parámetro $a h_{r}$, viene definido para ambientes urbanos (Hata, 1980), como:

$$
a h_{r}=3.20\left(\log _{10}\left(11.75 h_{m}\right)\right)^{2}-4.97 ; f>400 M H z
$$

Y para ambientes abiertos o sub-urbanos, 


$$
a h_{r}=\left(1.1 \log _{10}(f)-0.7\right) h_{m}-\left(1.56 \log _{10}(f)-0.8\right)
$$
2003).

Donde, $h_{m}$ es la altura de la antena del nodo móvil sobre el nivel del suelo (Anderson,

\section{Coeficiente de Regresión}

Basados en la ecuación 1, se derivan dos modelos que son el modelo de la ciudad de Oulu (Finlandia) y el modelo de la ciudad de Dortmund. Para el modelo Oulu se tiene un $P L\left(d_{0}\right)=128.95 \mathrm{~dB}$ con un valor de $n=2.32$. Para el modelo de Dortmund se tiene $P L\left(d_{0}\right)=132.25 \mathrm{~dB}$ con un valor de $n=2.65$, donde $n$ corresponde al gradiente distanciapotencia.

\section{Resultados y discusión}

Figura 5 muestra cada punto medido dentro de las instalaciones de la ESPOL, se los ha referenciado con nombres pertenecientes a circuitos tomados en las facultades correspondientes como son FIEC (color verde) y FCNM (color rojo). Se ha propuesto un modelo de pendientes múltiples debido a los diferentes ambientes que presenta el área de medición. Los puntos FIEC representan un ambiente cubierto por vegetación, mientras que los puntos FCNM representan un ambiente abierto/suburbano. Basado en estos resultados, el valor de la pendiente de los puntos FIEC es $-45.623 \mathrm{dBm} / \mathrm{m}$; mientras que, la pendiente de los puntos FCNM es $-58.264 \mathrm{dBm} / \mathrm{m}$.

\section{Figura 5}

\section{Mediciones del RSSI}

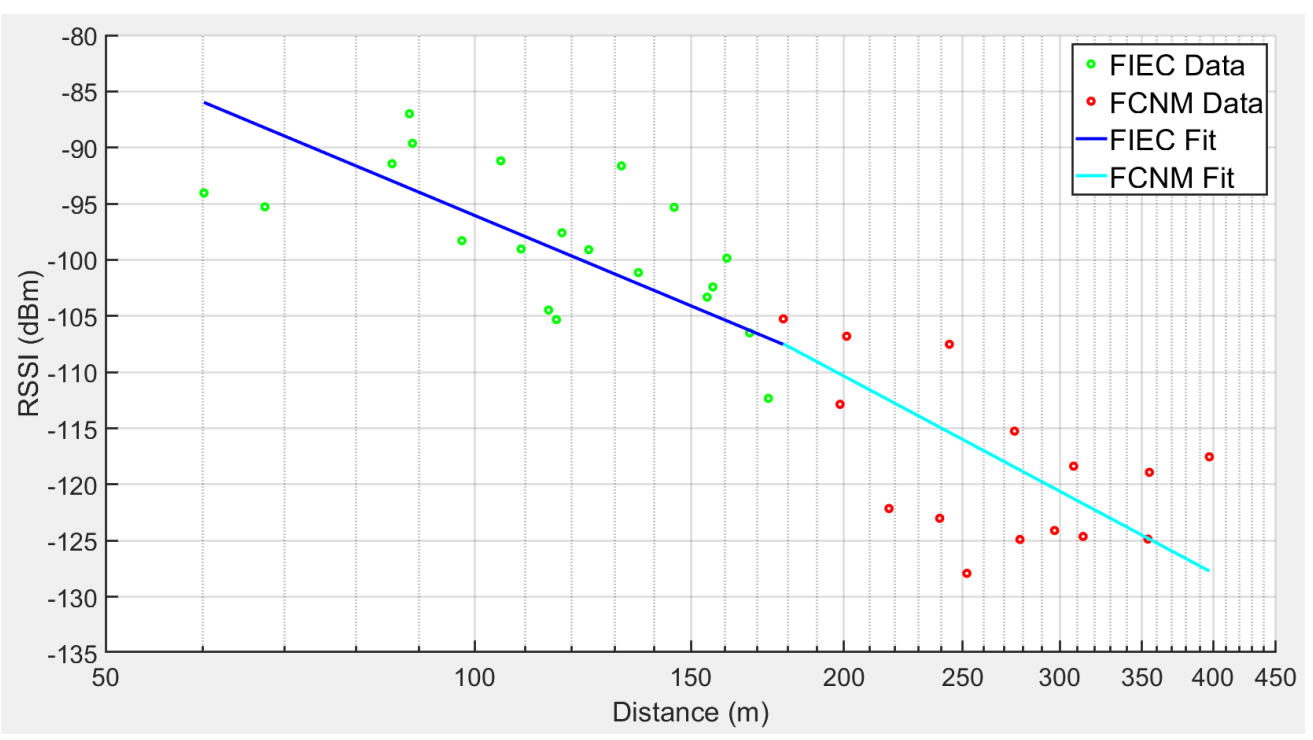

Figura 6 muestra la dispersión entre los datos, a través de un diagrama de cajas, donde se observa la variabilidad debido a los ambientes donde fueron tomados. Se observa que la media del RSSI de los datos FIEC se encuentra en $-98 \mathrm{dBm}$, con una predominancia de valores en el rango de -93 a $-103 \mathrm{dBm}$. Para los datos FCNM se tiene una media del RSSI igual a -120 $\mathrm{dBm}$, con un rango de datos comprendidos entre $-114 \mathrm{a}-125 \mathrm{dBm}$. Con este antecedente, se opta por la obtención de un modelo de gradiente múltiple para las pérdidas por propagación del modelo LoRa. 


\section{Figura 6}

Dispersión de datos según el ambiente

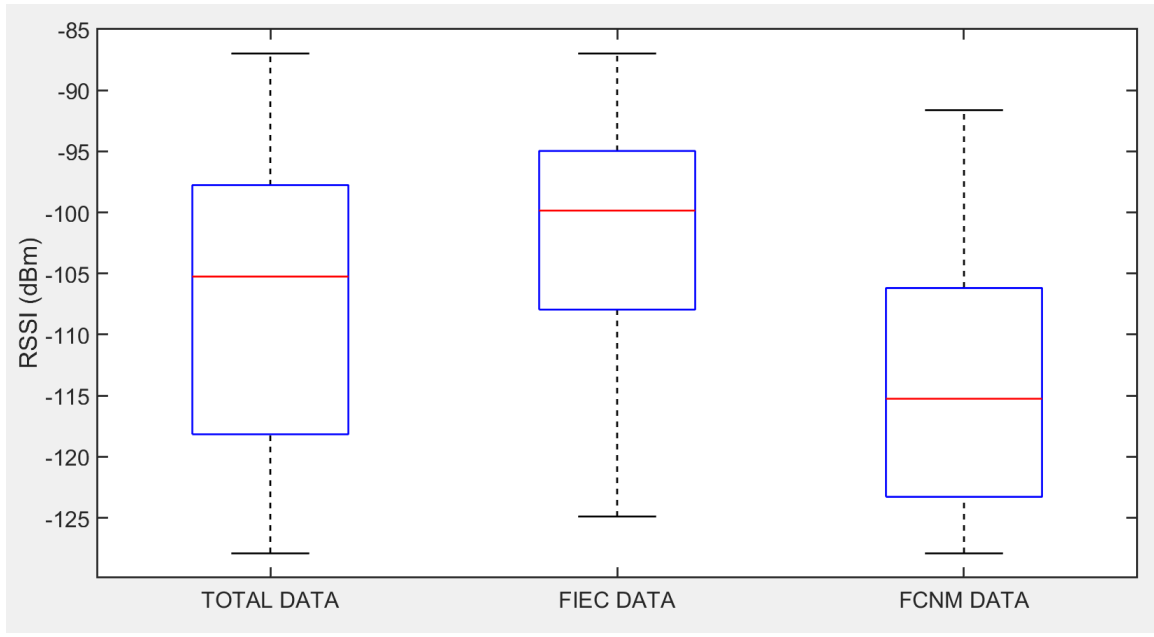

Basado en la intensidad de la señal recibida (RSSI), el cálculo de la pérdida por propagación viene dada por la fórmula:

$$
P L(R S S I)=|R S S I|+S N R+P_{T X}+G_{R X}
$$

Donde, SNR representa la medida señal-ruido captada por el dispositivo, $P_{T X}$ es la potencia efectiva isotrópica irradiada, $G_{R X}$ representa la ganancia de la antena usada en la recepción (Juha Petäjäjärvi, 2015). Figura 7 muestra el modelo de pérdida por propagación obtenido de las mediciones (línea cian), junto con sus dos gradientes $\propto_{1}=4.56$ para zonas cubiertas con vegetación y $\propto_{2}=5.83$ para espacios abiertos y/o suburbanos. Adicionalmente, se presenta en línea entre cortada roja el modelo de espacio libre, para tenerlo como referencia. Es importante recalcar que los resultados mostrados corresponden a la conexión de bajada (downlink) del nodo móvil, dada por el intercambio de paquetes entre nodos. También se muestran los modelos de Cost-231 (línea rosa), modelo Oulu (línea azul) y modelo Dortmund (línea verde).

\section{Figura 7}

Comparación de modelos por pérdidas en propagación

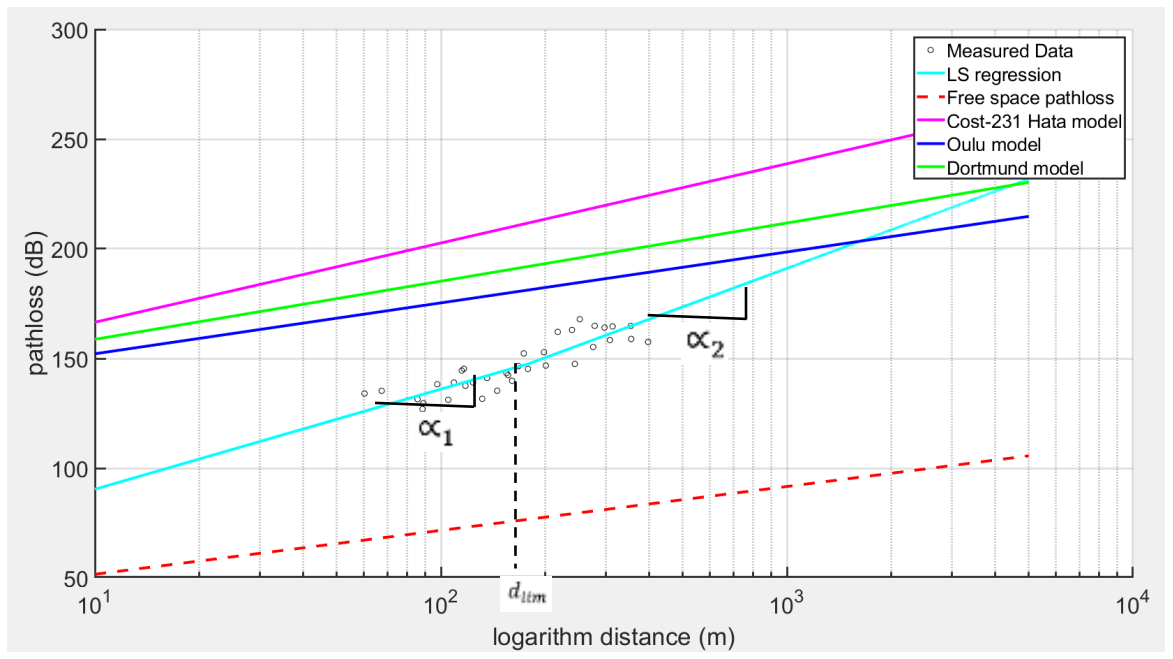


El modelo resultante obtenido viene dado por la ecuación:

$$
P L_{M}=P L_{0}+\left\{\begin{array}{l}
10 \propto_{1} \log _{10}(d) ; d<d_{\text {lim }} \\
10 \propto_{2} \log _{10}(d) ; d>d_{\text {lim }}
\end{array}\right.
$$

Donde, $P L_{0}$ corresponde a la pérdida de propagación de la señal recibida, dada por (6). $\propto_{1} \mathrm{y} \propto_{2}$ son los gradientes obtenidos, $\mathrm{d}$ es la distancia en metros y $d_{\text {lim }}=178.5$ metros.

Basado en el resultado del modelo obtenido, se calculó la desviación estándar $\sigma_{M}$ que existe, debido al debilitamiento por sombra (SF) para cada ambiente.

La Tabla 2 muestra el valor del gradiente obtenido para cada ambiente junto con su desviación estándar por debilitamiento de sombra.

\section{Tabla 2}

Desviación estándar de modelos para ambientes diferentes

\begin{tabular}{|c|c|c|}
\hline Ambiente & "n” o “ $\propto$ ” & $\boldsymbol{\sigma}_{M}(\mathbf{d B})$ \\
\hline Con vegetación (FIEC) & 4.56 & 5.1046 \\
\hline Abierto (FCNM) & 5.83 & 6.5246 \\
\hline
\end{tabular}

Se puede observar que el modelo empírico adoptado para comunicaciones inalámbricas COST-231 posee un mayor gradiente que el resto de modelos empíricos. El modelo obtenido en la ESPOL posee una divergencia con respecto a los modelos Oulu y Dortmund, debido a las condiciones físicas en que fueron tomados, ya que los dispositivos móviles se encontraban a diferentes alturas; adicionalmente, la presencia de edificios, zonas verdes que provocaban la ausencia de línea de vista en la mayoría de las mediciones y la variación del terreno.

Para la obtención de una métrica de comparación de las mediciones adquiridas, se calculó el error porcentual promedio "e", de los métodos en estudio de este artículo, mostrados en la Tabla 3.

Tabla 3

Error porcentual medio con respecto a modelo obtenido para distancias entre 10 a 5000 metros

\begin{tabular}{|c|c|}
\hline Modelo & $e=\frac{1}{N} \sum_{d=10}^{5000} \frac{\left|P L_{M}(d)-P L_{\text {modelo }}(d)\right|}{P L_{M}(d)} \chi 100$ \\
\hline FSL & $52.90 \%$ \\
\hline Oulu & $6.02 \%$ \\
\hline Dortmund & $6.85 \%$ \\
\hline Cost-231 & $21.03 \%$ \\
\hline
\end{tabular}

\section{Conclusiones}

Este artículo propone un modelo empírico de propagación que se evalúa con mediciones realizadas dentro del campus Prosperina de la ESPOL. Se obtiene un modelo de gradiente múltiple debido a la presencia de zonas con vegetación y zonas abiertas. 
Con los resultados obtenidos a través de mediciones y comparación de modelos, el modelo Oulu presenta menor error porcentual medio "e", con respecto al modelo de gradiente múltiple obtenido, como se muestra en la Tabla 3, en un rango de distancias comprendido entre 10 a 5000 metros.

La variación en las mediciones con los demás modelos se atribuye a la irregularidad que se tiene en el campus de la ESPOL y a la línea de vista existente o no al momento de realizarlas. Para futuras investigaciones se recomienda la utilización de circuitos en otras direcciones para tener un modelo más preciso sobre la pérdida de propagación dentro de este campus, y complementar el modelo gradiente para un ambiente cubierto de vegetación y otro al aire libre.

El ambiente cubierto por vegetación presenta un gradiente (pendiente) menor a las mediciones realizadas en espacio abierto en la FCNM, esto es debido a que existe mayor distancia, y la mayoría de puntos no poseen línea de vista.

Los modelos propuestos se desarrollan empleando una frecuencia de $915 \mathrm{MHz}$, que es la que utiliza el estándar LoRa, para futuras investigaciones se puede establecer un modelo con otra tecnología LPWAN como Sigfox, ya que trabaja en la misma banda no licenciada, para establecer diferencias y comparación entre modelos.

Otro aspecto importante de esta investigación es que se realiza en la estación climática de invierno, con días soleados, por lo que posiblemente otros factores como la temperatura, horario e incluso estación de verano, podrían afectar el modelo propuesto.

\section{Reconocimientos}

Este trabajo ha sido financiado parcialmente por CEDIA, a través del Grupo de Trabajo de IoT y Ciudades Inteligentes.

Los autores desean expresar su agradecimiento a la Escuela Superior Politécnica del Litoral (ESPOL) por la prestación y pronta gestión de permisos para acceder y hacer uso de sus instalaciones.

\section{Referencias}

Anderson, H. R. (2003). Fixed Broadband Wireless System Design. Wiley.

Ayanle I. Ali, S. Z. (2019). ZigBee and LoRa based Wireless Sensors for Smart Environment and IoT Applications. Global Power, Energy and Communication Conference. Cappadocia.

Commission), D.-G. f. (1999). Digital mobile radio towards future generation systems: Final Report. EUR.

Elmustafa Sayed Ali Ahmed, M. E. (2019). Internet of things in Smart Environment: Concept, Applications, Challenges, and Future Directions. World Scientific News.

Gutierrez, S. M. (2019). Smart Mobile LoRa Agriculture System based on Internet of Things. IEEE 39th Central America and Panama Convention.

Hata, M. (1980). Empirical formula for propagation loss in land mobile radio services. En IEEE Transactions on Vehicular Technology (págs. 317-325). IEEE.

Hendrik Linka, M. R. (2018). Path Loss Models for Low-Power Wide-Area Networks: Experimental Results using LoRa. VDE ITG-Fachbericht Mobilkommunikation. Osnabrück. 
Juha Petäjäjärvi, K. M. (2015). On the Coverage of LPWANs: Range Evaluation and Channel Attenuation Model for LoRa Technology. 14th International Conference on ITS Telecommunications (ITST). Copenhagen, Denmark.

Kais Mekki, E. B. (2018). A comparative study of LPWAN technologies for large-scale IoT. En ICT Express.

Luis F. Ugarte, M. C. (2019). LoRa Communication as a Solution for Real-Time Monitoring of IoT Devices at UNICAMP. International Conference on Smart Energy Systems and Technologies (SEST).

Massimo Ballerini, S. T. (2020). NB-IoT vs. LoRaWAN: An Experimental Evaluation for Industrial Applications. IEEE Transactions on Industrial Informatics.

Oratile Khutsoane, B. I.-M. (2017). IoT Devices and Applications based on LoRa/LoRaWAN.

Philip J. Basford, F. M.-C. (2020). LoRaWAN for Smart City IoT Deployments: A Long Term Evaluation. En Sensors.

Usman Raza, P. K. (2017). Low Power Wide Area Networks: An Overview. IEEE Communications Surveys \& Tutorials.

V.S. Abhayawardhana, I. W. (2005). Comparison of empirical propagation path loss models for fixed wireless access systems. 2005 IEEE 61st Vehicular Technology Conference. Stockholm, Sweden.

Wael Ayoub, A. E.-C. (2020). Media Independent Solution for Mobility Management in Heterogeneous LPWAN Technologies. Computer Networks. 